OPEN ACCESS

Edited by:

Błazej Misiak,

Wroclaw Medical University, Poland

Reviewed by:

Marina Šagud

University of Zagreb, Croatia

Letitia Travaglini,

United States Department of

Veterans Affairs, United States

*Correspondence:

Alistair Lum

alistair.lum@newcastle.edu.au

Specialty section This article was submitted to Schizophrenia, a section of the journal

Frontiers in Psychiatry

Received: 07 August 2018 Accepted: 18 October 2018 Published: 06 November 2018

Citation:

Lum A, Skelton E, Wynne O and Bonevski B (2018) A Systematic Review of Psychosocial Barriers and Facilitators to Smoking Cessation in People Living With Schizophrenia. Front. Psychiatry 9:565. doi: 10.3389/fpsyt.2018.00565

\section{A Systematic Review of Psychosocial Barriers and Facilitators to Smoking Cessation in People Living With Schizophrenia}

\author{
Alistair Lum*, Eliza Skelton, Olivia Wynne and Billie Bonevski \\ School of Medicine and Public Health, University of Newcastle, Newcastle, NSW, Australia
}

Background: People living with schizophrenia are less likely to quit smoking compared with the general population and people living with other psychiatric disorders. Understanding the schizophrenia-specific psychosocial barriers and facilitators to smoking cessation is important for designing effective smoking cessation interventions. We aimed to systematically review research examining psychosocial barriers and facilitators to smoking cessation in people living with schizophrenia.

Methods: We followed the PRISMA statement to conduct a systematic literature review examining psychosocial barriers and facilitators to smoking cessation in people living with schizophrenia. We searched EMBASE, Medline, PsycINFO, and CINAHL databases from inception to 14 June 2018 to identify relevant articles. We included peer-reviewed original research articles that examined psychosocial barriers and facilitators to smoking cessation, as well as factors associated with maintenance of smoking habits in people living with schizophrenia spectrum disorders. Qualitative, quantitative, or mixed-methods study designs were included. Three authors screened titles, abstracts, and full-texts using the eligibility criteria. We conducted a narrative synthesis of the data to account for the heterogeneity of study designs. We analyzed qualitative and quantitative studies separately.

Results: We identified 685 studies from our systematic search and screened the full-text of 134 articles. The final set of 23 articles included 20 quantitative studies and 3 qualitative studies. The most commonly cited barrier to smoking cessation in people living with schizophrenia was cravings and addiction, followed by a perceived increased risk of negative affect associated with quitting smoking. People living with schizophrenia reported smoking to manage stress and to maintain social relationships. People living with schizophrenia were found to be less likely to receive cessation support from health professionals than smokers without schizophrenia. Health concerns were the most commonly mentioned facilitator to quit smoking.

Conclusions: People living with schizophrenia experience a wide range of barriers to smoking cessation. The influence of these barriers on smoking cessation likelihood 
may be greater among people living with schizophrenia than people without psychiatric disorders. Health professionals play an important role in smoking cessation for people living with schizophrenia and should consider barriers and facilitators identified in this review to support quitting in this vulnerable population.

Keywords: systematic review, barriers, facilitators, smoking cessation, schizophrenia, psychosocial

\section{INTRODUCTION}

Approximately $65 \%$ of people living with schizophrenia smoke cigarettes $(1,2)$. People living with schizophrenia are significantly less likely to quit smoking compared with the general population and those living with other psychiatric disorders, such as bipolar disorder and depression (1). Given the elevated prevalence of tobacco smoking and low cessation rates, people living with schizophrenia are at higher risk of developing smokingrelated malignancies, cardiovascular disease, and respiratory disease and are more likely to experience premature mortality than the general population (3). Improving smoking cessation rates in people living with schizophrenia will be integral to improving the health of this vulnerable population. Clinical Practice Guidelines for the treatment of tobacco smoking suggest clinicians provide a dual approach consisting of both pharmacological (e.g., bupropion, varenicline, and nicotine replacement therapy [NRT]) and psychosocial (e.g., cognitive behavioral therapy $[\mathrm{CBT}]$, motivational interviewing $[\mathrm{MI}$, information and education interventions, and social support) strategies for people living with schizophrenia (4-6).

Research examining the pharmacological treatments for tobacco smoking among people living with schizophrenia exceeds the quantity of corresponding psychosocial research, potentially limiting the depth and breadth of smoking cessation advice that clinicians can provide. Pharmacological interventions appear to be effective with few safety concerns. A meta-analysis of five trials comparing bupropion or bupropion and NRT with placebos or placebos with NRT found that participants in the bupropion group were almost three times more likely to abstain from smoking at 6 month follow-up compared with those in the placebo group (7). Two trials that compared varenicline with placebo found almost five times greater smoking cessation rates in the varenicline group at end of treatment (7). Pharmacological smoking cessation interventions appear to be appropriate for people living with schizophrenia, however the percentage of successful quitters is reportedly small, between 12 and 19\% (7).

In line with clinical guidelines, many randomized clinical trials of pharmacological interventions were delivered alongside psychosocial strategies for treatment of tobacco smoking (7). Yet, few trials assessed the benefits associated with psychosocial strategies in these combined interventions. The limited number of combined trials have shown limited long-term effect. One such trial compared a smoking cessation program founded on CBT and MI principles plus NRT with usual care plus NRT, and found higher smoking reduction rates in the intervention group at 3 months, but no differences in smoking abstinence or reduction rates beyond 6 months (8). Contingency reinforcement using money with or without NRT or bupropion have been associated with higher smoking abstinence compared with a minimal intervention group (9) and smoking reduction rates compared with a pharmacotherapy only group (10), however long-term effects were not reported.

Very few randomized controlled trials of psychosocial interventions for smoking cessation or reduction in people living with schizophrenia have been conducted (11-14). Psychosocial programs for smoking cessation in people living with schizophrenia have used a variety of approaches, including psychoeducation, MI, CBT, social skills training, relapse prevention, monetary contingent reinforcement strategies, or a combination of these approaches (15). One study compared a high-intensity program incorporating MI, social skills training, NRT education, and relapse prevention with a moderateintensity program focused on medication compliance and NRT education (13). Smoking cessation rates did not differ between groups, with $21 \%$ abstinent at 12 -weeks after the target quit date, $17 \%$ at 6 month follow-up, and $14 \%$ at 12 month follow-up. A comparison of the American Lung Association (ALA) smoking cessation program with a schizophrenia-targeted program comprised of MI, psychoeducation, and relapse prevention strategies found significantly higher abstinence rates in the ALA group at 6-month follow-up (11). The 6 month smoking cessation rates of the psychosocial interventions described above range between 11 and $18 \%(11,13)$, which are comparable to rates achieved in pharmacotherapy trials, which range between 12 and $19 \%$ (16-18).

Research examining the neurobiological factors associated with smoking maintenance among people living with schizophrenia is substantial and continues to grow (19). This research is helping inform advances in pharmacological treatment options (19). In contrast, the theory underlying psychosocial interventions for people living with schizophrenia has not been well-defined in the literature, which may limit the effectiveness of psychosocial interventions for smoking cessation for people living with schizophrenia. Ziedonis and George (20) reviewed the literature on smoking cessation and schizophrenia prior to the development and evaluation of a psychosocial intervention promoting smoking cessation in people living with schizophrenia. However, their review of neurobiological and clinical issues failed to address psychosocial factors associated with smoking cessation consistent with their psychosocial intervention. Steinberg and Williams (21) examined the necessary modifications to treatment components of smoking cessation programs to better match the needs of people living with schizophrenia. They found that people living with schizophrenia may require more intervention sessions or sessions over a longer duration, content delivery adaptations to account for neurocognitive deficits common in people living 
with schizophrenia and social skills training (21). While these findings are important, this examination of intervention factors does not account for barriers of smoking cessation in people living with schizophrenia identified in the non-intervention literature.

Current reviews on barriers to smoking cessation in people living with schizophrenia have included people with other mental illnesses, such as bipolar disorder or severe depression. Common individual barriers to smoking cessation identified in these reviews include the desire to manage stress and avoid withdrawal symptoms, and the belief that smoking provides a sense of identity. Many people living with mental illness are not given cessation support from health care providers $(22,23)$. Smoking tobacco is often socially accepted among people living with mental illness $(23,24)$. Current evidence on facilitators of smoking cessation among people with schizophrenia, including perceived health, and financial benefits and social support for quitting, are also commonly experienced by people with other mental illnesses and the general population $(23,25)$.

Schizophrenia-specific barriers and facilitators to smoking cessation are less well-understood than those affecting people with all mental illness. One commonly mentioned reason for smoking among people with schizophrenia is the desire to manage negative symptoms $(23,24,26)$. Managing negative symptoms, such as negative affect, anhedonia, and loss of motivation, can improve social and vocational functioning among people living with schizophrenia (27). While this self-medication hypothesis is recognized as a schizophrenia specific barrier to smoking cessation, continued efforts to determine other barriers and facilitators are required to ensure comprehensive support is available (28).

A stronger understanding of schizophrenia-specific barriers and facilitators to smoking cessation, such as reduction of negative symptoms, is required to help clinicians to provide optimal treatment options and intervention developers to better tailor their programs to the unique needs of people living with schizophrenia (28-31). Currently, psychosocial interventions have been developed without consideration of the full range of psychosocial barriers and facilitators. Thus, we cannot be confident that current interventions adequately address the unique psychosocial factors contributing to smoking cessation in people living with schizophrenia. Therefore, we aimed to systematically review research examining the psychosocial barriers and facilitators to smoking cessation in people living with schizophrenia. We asked two research questions:

1. What psychosocial barriers and facilitators affect smoking cessation in people living with schizophrenia?

2. Do people living with schizophrenia experience more psychosocial barriers that affect smoking cessation than people without mental illness?

\section{METHODS}

\section{Design}

We systematically reviewed original research examining psychosocial barriers and facilitators to smoking cessation in people living with schizophrenia. The PRISMA statement guided the conduct of the review (32). We used the Covidence software in screening articles (33). We registered the review with Prospero (CRD42018103332).

\section{Search Strategy}

We searched EMBASE, Medline, PsycINFO, and CINAHL databases using keywords from inception to 14 June 2018 to identify relevant articles. Our search terms were [smoking OR tobacco OR cigarette OR nicotine OR e-cig] AND [schizophrenia OR psychosis OR schizoaffective OR schizophreniform OR delusional disorder OR psychotic OR psychoses] AND [factor\$ OR determinant\$ OR variable\$ OR covariable\$ OR predictor\$ OR barrier\$ OR facilitator\$] AND [smoking cessation OR quitting smoking OR abstinence OR withdrawal OR quit\$]. \$ indicated truncation.

\section{Eligibility}

We included peer-reviewed original research articles that examined psychosocial barriers and facilitators to smoking cessation, as well as factors associated with maintenance of smoking habits, in people living with schizophrenia spectrum disorders. Articles that included mixed diagnosis samples composed of $50 \%$ or more of participants with schizophrenia were included. We included participants living with schizophrenia as well as healthcare providers working with people living with schizophrenia in $50 \%$ or more of their cases. We included articles that reported outcomes relating to people who identify as smokers without a requirement that the study define the smoking status of participants. This inclusive approach was designed to increase the number of studies included in our review. We included qualitative, quantitative, and mixed-methods study designs. Cross-sectional and longitudinal quantitative study designs with or without a comparison group were eligible for this review. We chose to include studies with baseline data from intervention trials to increase the amount and quality of relevant data, yet the results must be interpreted with caution due to the potential bias associated with recruitment into an intervention trial. Articles published in gray literature or in languages other than English were excluded.

\section{Study Selection}

Two authors (AL and ES) simultaneously screened titles and abstracts using the eligibility criteria. All articles not excluded were screened using the full text by one author (AL), with twothirds of articles screened by a second author (ES or OW). These three authors discussed any conflicts in screening.

\section{Data Extraction}

One author (AL) extracted data from all included articles using a standardized pre-piloted data extraction form. Data extracted included age, sex, study design, country of study, sample size, control group characteristics, diagnostic characteristics (e.g., recruitment site, percentage of sample which was diagnosed with schizophrenia, and criteria to assess diagnosis), smoking characteristics (i.e., number of cigarettes smoked daily, age 
smoking commenced, tool to assess smoking status, and nicotine dependence), barrier and facilitators characteristics (i.e., maintenance factors relating to smoking or barriers or facilitators to smoking cessation, assessment tool to measure factors, and relationship to outcome measure) and outcome measure (i.e., type of smoking outcome and assessment tool of outcome).

\section{Quality Appraisal}

One author (AL) critically appraised the risk of bias in all articles using the QualSyst tool (34). The QualSyst tool was developed as a tool designed to measure the risk of bias in a range of studies, including randomized trials and quantitative, qualitative, and mixed methods studies. Two checklists with manuals to guide scoring are available; one for quantitative studies with 14 items and one for qualitative studies with 10 items. We report the percentage of checklist items met for all studies to improve interpretability, with higher percentages indicating lower risk of bias.

\section{Data Analysis}

We conducted a narrative synthesis of the data to account for the heterogeneity of study designs. We synthesized data on barriers and facilitators to quitting and motivators to smoke. We analyzed qualitative and quantitative studies separately. Due to the lack of theory underlying psychosocial barriers and facilitators to smoking cessation in people living with schizophrenia, we took an inductive approach to data synthesis.

\section{RESULTS}

\section{Search Results}

We identified 685 studies from our systematic search, of which 14 were duplicates. We removed 537 of the 671 articles based on titles and abstracts that indicated the article was not relevant to our aims. We screened the full-text of the remaining 134 articles, and removed a further 111. The main reasons for excluding articles at the full-text screening stage were that the article did not examine psychosocial barriers or facilitators to smoking cessation, did not examine smoking cessation, did not present original data, or did not include a sample comprised of at least $50 \%$ of participants with schizophrenia (see Figure 1). The final set of articles included 23 articles, of which 20 had quantitative designs and 3 had qualitative designs.

\section{Study Characteristics}

Of the 3,557 participants in included studies, 3,257 had a diagnosis of schizophrenia (91.6\%). All articles were published between 1996 and 2017. Eleven (48\%) articles were published in the United States, five (22\%) in Australia, three (13\%) in Canada and one each in Turkey, Greece, Israel, the United Kingdom, and Scotland (4\%). Of the 20 quantitative studies, 12 (60\%) had cross-sectional designs, 6 (30\%) examined baseline data from intervention trials, 1 (5\%) was a non-randomized within-group trials, and 1 (5\%) was a non-randomized controlled trial.

The quantitative articles met between 50 and $88 \%$ (median $=80 \%$ ) of the QualSyst risk of bias criteria, while

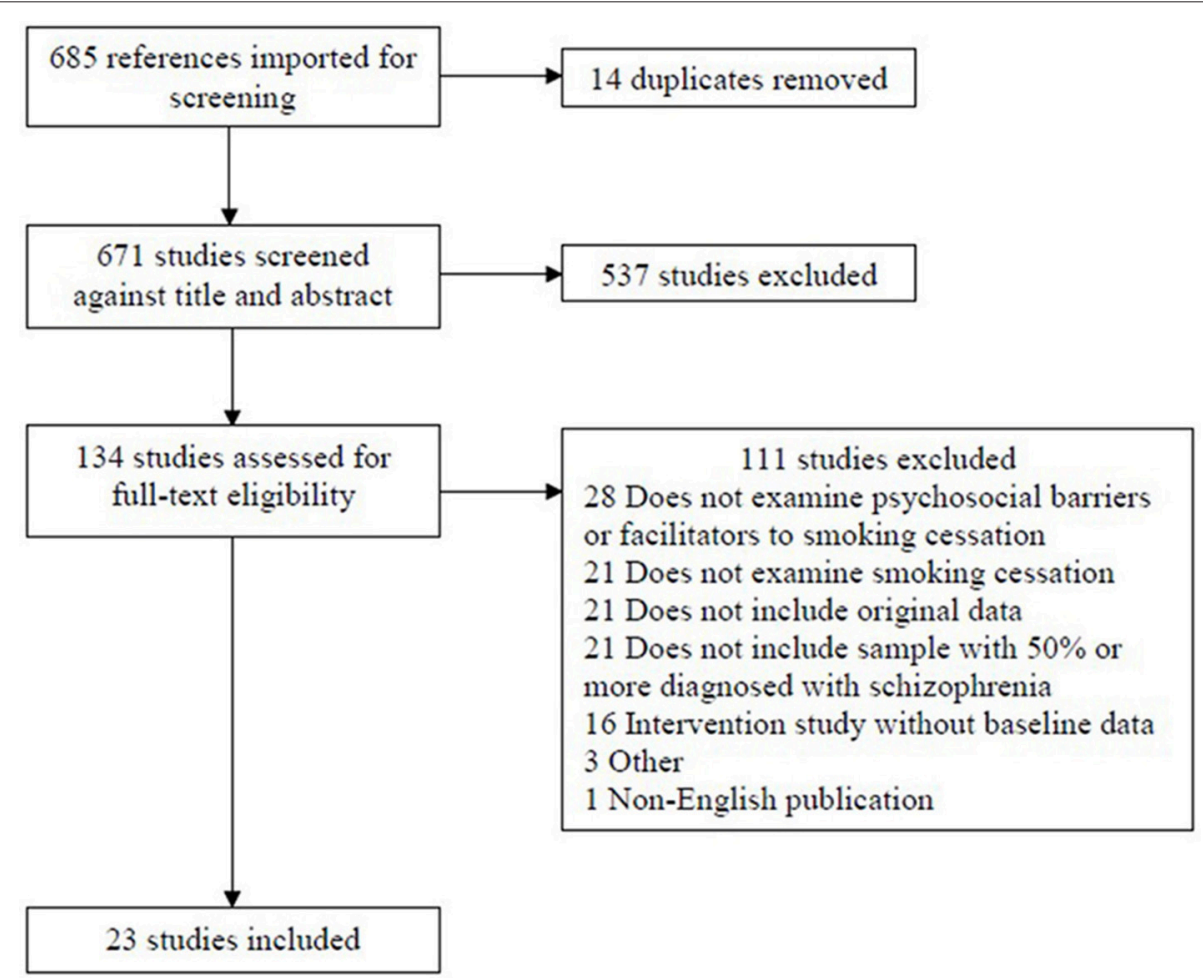

FIGURE 1 | Flow diagram of screening process and outcomes. 
the qualitative articles met between 45 and $85 \%$ of the criteria (median $=85 \%)$. Seventy percent of quantitative $(14 / 20)$ and $67 \%$ of qualitative $(2 / 3)$ studies met $75 \%$ or more of the QualSyst criteria. These outcomes indicate that the combined data has a low risk of bias and the findings can be considered as reliable indicators of barriers and facilitators to smoking cessation in people living with schizophrenia. The majority of quantitative studies met the QualSyst criteria of reporting study objectives, sufficiently disclosing participant characteristics, recruiting an appropriate sample size, describing methods of data analysis, reporting an estimate of variance, reporting results in sufficient details, and drawing conclusions supported by the results. Outcome variables and method of participant selection were inconsistently reported with sufficient detail and study design was often not explicitly reported. The context of the study, sampling strategy, data analysis techniques, and coding biases were only partially described in at least two of the three qualitative studies. Study and participant characteristics, QualSyst scores, and a summary of key study outcomes are presented in Table 1 for quantitative studies (see sections Cravings and Addiction, To Reduce Negative Affect, Social Facilitation, Stress Management, Concern for Health Risks, Physician Advice to Quit Smoking, Systemic Barriers, Social Pressure to Quit, and Additional Barriers and Facilitators to Smoking Cessation) and Table 2 for qualitative studies (see section Qualitative Findings).

\section{Cravings and Addiction}

The most commonly cited barrier to smoking cessation in people living with schizophrenia was cravings and addiction (nine studies). Two quantitative studies examining concerns associated with quitting found that cravings and addiction were the highest reported risks associated with smoking cessation $(35,36)$. Cravings were also frequently reported as a concern related to smoking cessation in two studies $(37,38)$, however one study indicated that cravings were not perceived as a reason for people living with schizophrenia to smoke cigarettes (39). Perceived or actual cravings associated with smoking abstinence were significantly higher among people living with schizophrenia compared with people without mental illness in three studies $(36,40,41)$, yet were similar across groups in two studies $(42,43)$. One of the two studies in which cravings were reportedly higher in people living with schizophrenia than people without mental illness found that cravings increased over $72 \mathrm{~h}$ of abstinence in people living with and without schizophrenia, with no difference in the rate of increase across groups (41). Three studies examining whether sex and age was associated with cravings found no association $(40,42,43)$. Two of the three studies which found higher perceived risk of cravings in people living with schizophrenia were based on small group sizes of 18 and 28 .

\section{To Reduce Negative Affect}

A perceived increased risk of negative affect associated with quitting smoking was examined in seven studies. Perceived risk of increased negative affect was higher in people living with schizophrenia compared with people without mental illness in two studies $(41,43)$ and equal in three studies $(36,42,44)$.
One study reported that $31 \%$ of people living with schizophrenia smoked to reduce symptoms of anxiety and depression (45), while another reported that reducing negative affect would be the strongest motivator to smoke during abstinence (38). Filia et al. (43) found that females reported significantly higher perceived risk of negative affect associated with quitting compared with males.

\section{Social Facilitation}

Seven studies examined the relationship between smoking cessation and social facilitation in people living with schizophrenia. One study found that people living with schizophrenia were more likely to smoke to improve social functioning than people without mental illness (42). Another study found that people with schizoaffective disorder reported that positive social effects were related to lower intention to quit; a relationship that was not identified in people living with schizophrenia or without mental illness (44). One study reported that people living with schizophrenia felt equally as likely as people without schizophrenia to be socially ostracized if they were to quit smoking (43). Non-comparison studies also indicated that smoking was an important factor contributing to social comfort. One study found social factors were considered to be the second strongest factor contributing to smoking temptations in people living with schizophrenia (38). Krishnadas et al. (45) reported that $14 \%$ of respondents reported smoking to socialize better, while Kourakos and Koukia (46) found that $83 \%$ of psychiatric inpatients with schizophrenia believed that visitors should be allowed to smoke with patients. Neither age (42) nor did sex (43) appear to be associated with perceived social facilitation as a motivator to smoke.

\section{Stress Management}

Five studies reported on the impact of smoking on stress reduction in relation to smoking cessation. One study reported that people living with schizophrenia were significantly more likely to smoke to reduce stress compared with people from the general population (40). A second study found no differences in ratings of stress as a concern related to quitting smoking between people living with schizophrenia and people without mental illness (36). However, the small group size of people living with schizophrenia may have reduced the likelihood of identifying true group differences (i.e., Type 2 error). Two other studies identified stress reduction as the main reason for smoking $(35,37)$, while another found that $60 \%$ of people living with schizophrenia smoke to relax (45). Two studies found that sex and age were not associated with stress management as a motivator to smoke $(40,42)$.

\section{Concern for Health Risks}

We identified eight studies examining perceptions of health risks as facilitators to smoking cessation in people living with schizophrenia. In four of these studies, people living with schizophrenia reported perceptions that the health benefits associated with quitting smoking were equal to perceptions of people without mental illness $(36,40,43,44)$. One included study reported that concern for health was the highest rated 


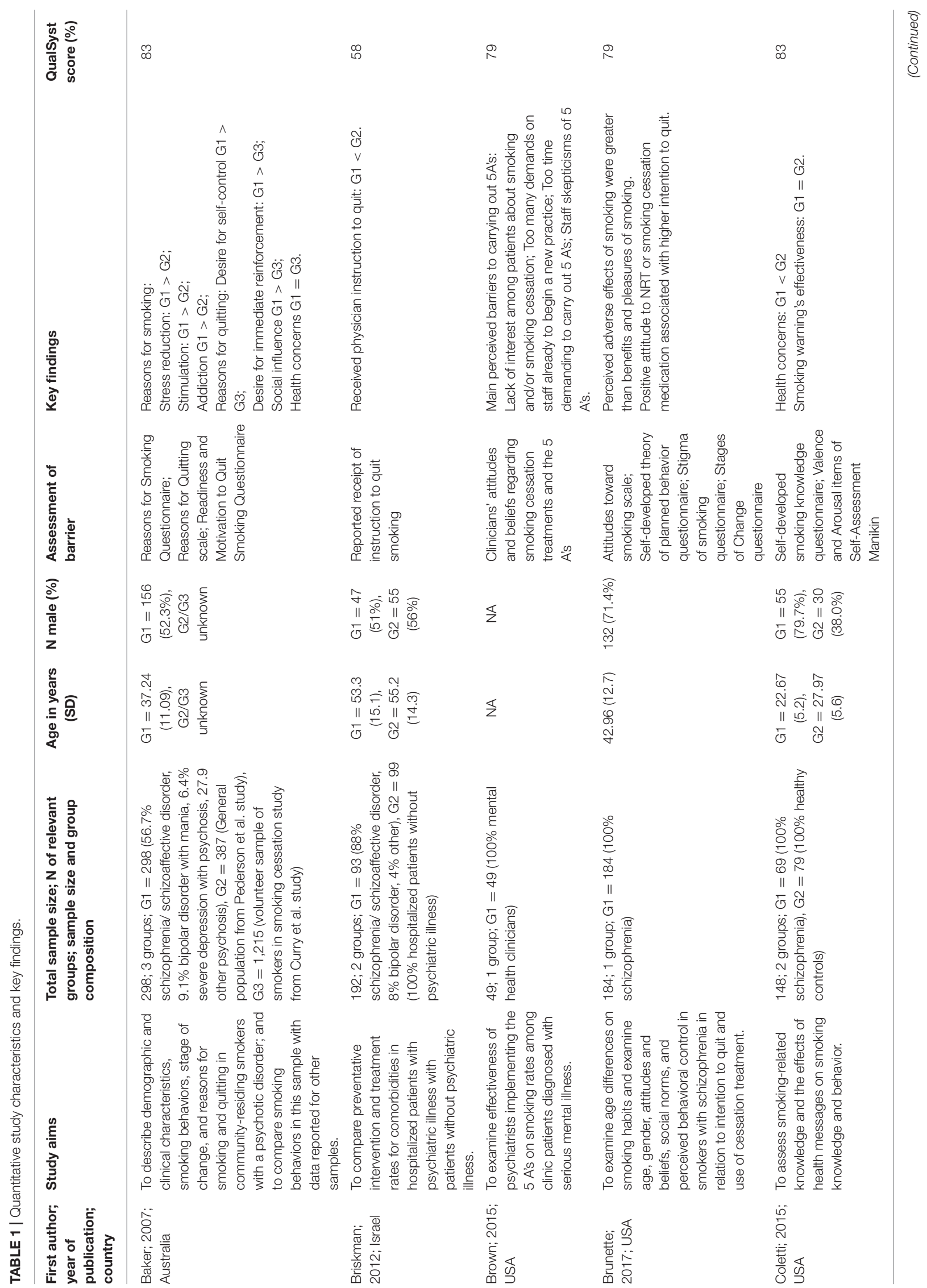




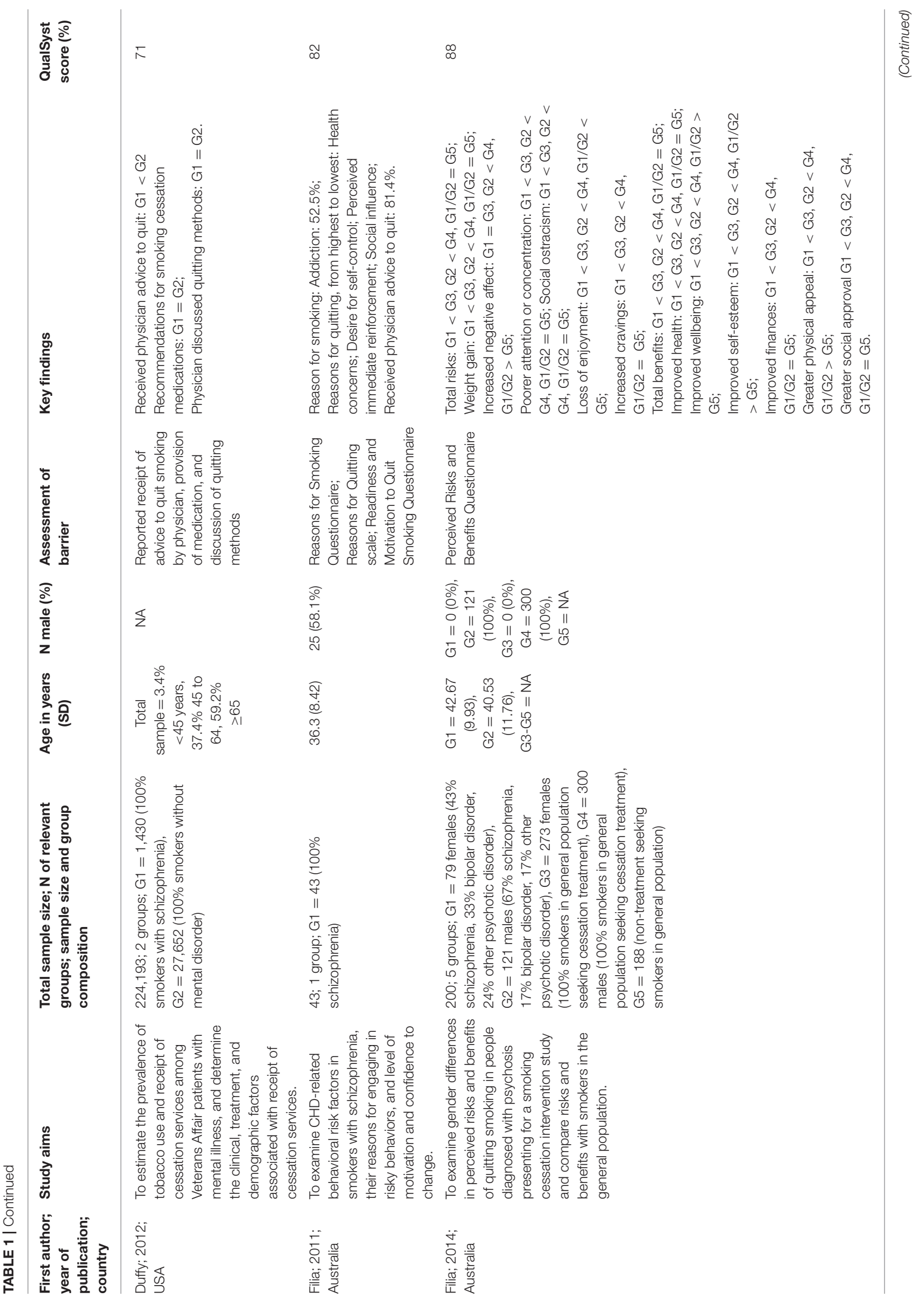




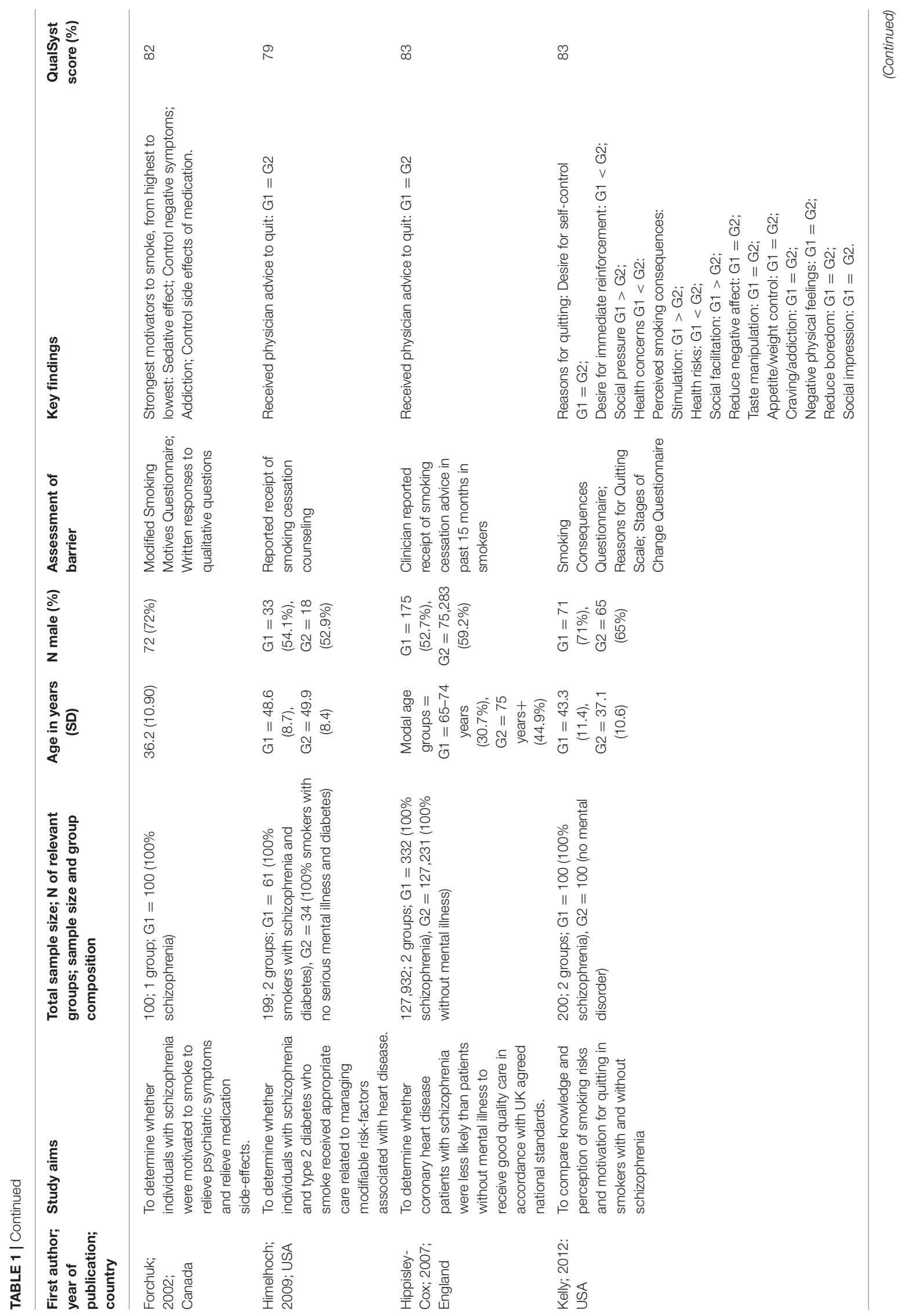




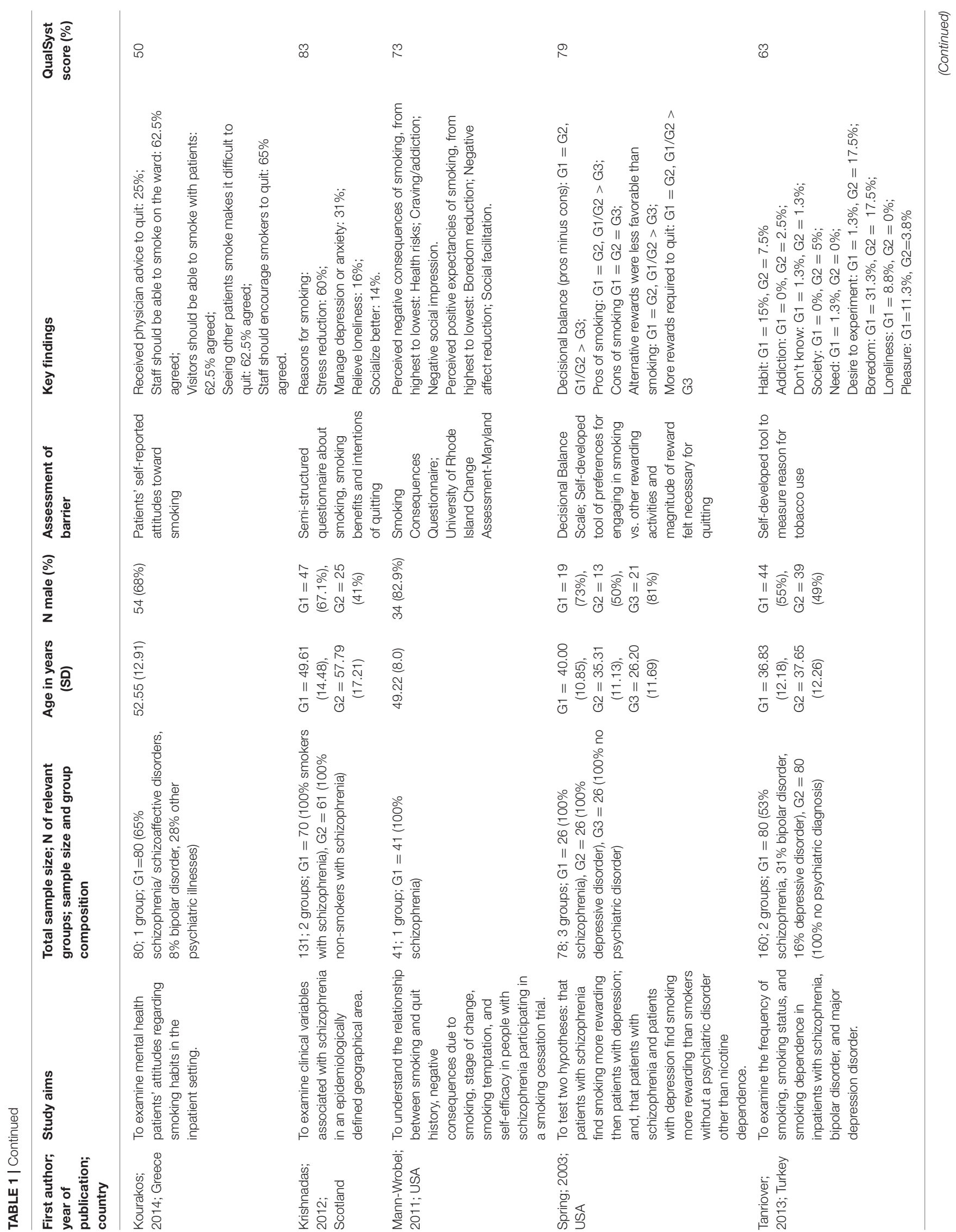




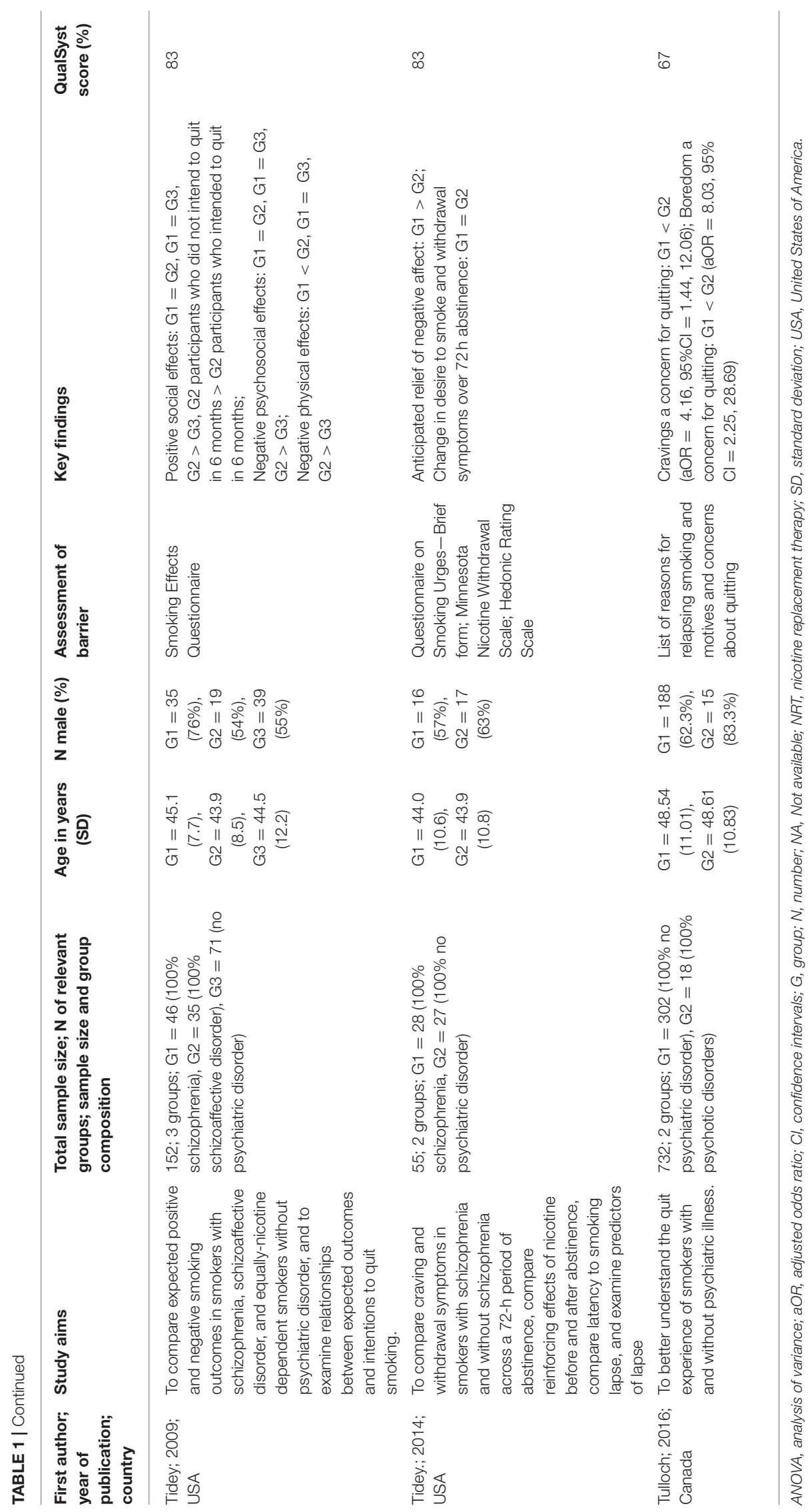




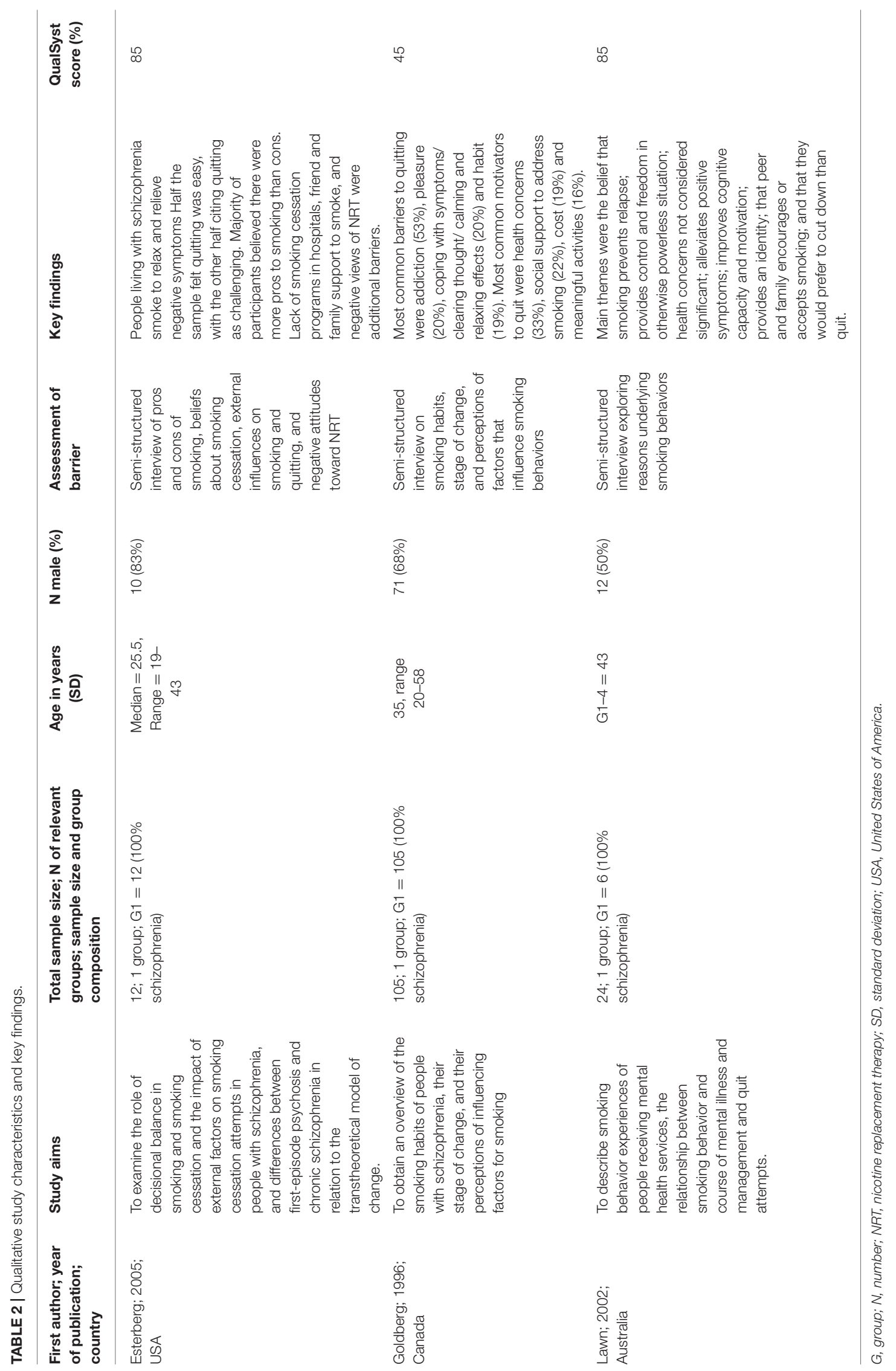


reason to quit smoking (35) and another study reported that people living with schizophrenia perceived smoking-related health implications were the most negative consequences of smoking (38). However, two studies found that people living with schizophrenia reported lower health-related concerns than people without mental illness $(42,47)$. A greater proportion of people with recent onset psychosis compared with smokers without mental illness believed smoking had fewer adverse health effects, including for stroke, brain damage, lung disease, heart disease, cancer, and miscarriage (47). No differences in concern for health between males and females or relating to age were identified $(40,42,43)$.

One study compared picture and video health warnings on perceived effectiveness and emotional valence and arousal in people living with schizophrenia and people without mental illness (47). People living with schizophrenia perceived the health warnings as more effective than controls. Perceived effectiveness of health warnings was positively associated with the emotional valence and arousal reported by participants.

\section{Physician Advice to Quit Smoking}

Seven studies reported on advice to quit by a health professional. One study reported that people living with schizophrenia were less likely to have received instruction for smoking cessation than people without mental illness (48), two found equal levels of advice provided to people living with and without schizophrenia $(49,50)$, while a fourth study found mixed results depending on the type of advice provided (51). The median percentage of people living with schizophrenia reporting receipt of advice to quit by a health professional was $80 \%$, with a range between 25 and $94 \%(35,46,49-51)$, while the median for people without schizophrenia was $83 \%$, ranging from 79 to $98 \%$ (49-51). Four studies comparing rates of physician advice were conducted in samples with smoking-related comorbidities, such as cardiovascular heart disease or diabetes, or in unique populations, such as members of Veterans' Affairs.

Brown et al. (52) examined the perceptions of mental health clinicians in providing the " 5 A's" (ask, advise, assess, assist, arrange) of smoking cessation advice to their patients, which included a majority percentage of people living with schizophrenia. Clinicians rated their perceived lack of interest among patients of all diagnoses to discuss smoking and/or smoking cessation, too many demands on staff already to begin a new practice, too time demanding to carry out 5 A's, and staff skepticisms about the value of the 5 A's as the strongest barriers preventing implementation of the 5 A's.

\section{Systemic Barriers}

Patients with schizophrenia in a psychiatric inpatient unit with few rules regarding smoking on the ward were generally supportive of the hospital's smoking policy (46). Seventy-five per cent agreed that the liberal ward rules about smoking were correct, while $63 \%$ believed that visitors and/or staff should be allowed to smoke on the ward. However, 90\% of participants reported that it was too difficult to quit, with $63 \%$ believing that seeing other patients smoke would make it difficult to quit and $65 \%$ reporting that staff should set a good example.

\section{Social Pressure to Quit}

Two studies identified social pressures to quit smoking as reportedly higher among people living with schizophrenia compared with people without mental illness $(40,42)$, however another two studies found equal levels of social pressure across groups $(36,43)$. No study identified a link between age or sex and social pressure to quit among people living with schizophrenia $(40,42)$. One study also reported that people living with schizophrenia who perceived their friends would be more likely to approve of NRTs or smoking cessation medication had higher intentions to quit smoking using these two pharmacotherapies (53).

\section{Additional Barriers and Facilitators to Smoking Cessation}

Reduction of boredom was another common reason people living with schizophrenia smoke. Five studies examined boredom as a reason to smoke, with one study finding people living with schizophrenia were eight times more likely than people without mental illness to report boredom as the reason for smoking (36). Three studies reported that boredom was the highest rated reason that people living with schizophrenia smoked cigarettes $(38,39,42)$. Yet one of these studies found no difference between people living with schizophrenia and people without mental illness in ratings of boredom as a reason to smoke cigarettes (42).

Five studies examined stimulation as a reason to smoke, with two studies identifying people living with schizophrenia as significantly more likely to smoke for stimulation or arousal when compared with people without mental illness $(40,42)$. Two studies found no difference between people living with and without schizophrenia $(43,44)$. Stimulation as a reason to smoke was not associated with age or sex (40, 42).

People living with schizophrenia were equally as likely as people without mental illness to smoke for the purpose of managing weight in four studies (36, 42-44). Mann-Wrobel et al. (38) reported prevention of weight gain as the least important positive consequence of smoking among people living with schizophrenia. One study found that females were more likely than men to report higher perceived risk of weight gain associated with quitting (43).

One study compared perceptions of the pros and cons of smoking in three groups comprised separately of people living with schizophrenia, depression, or without mental illness (54). People living with schizophrenia and depression reported similar levels of pros and cons of smoking, with the combined schizophrenia and depression group reporting significantly more pros of smoking than the control group and an equal number of cons. The combined schizophrenia and depression group also reported that smoking was more rewarding than a higher number of alternative pleasurable activities than the control group. People living with schizophrenia reported requiring more rewards, such as coffee or money, to quit smoking than people without a mental illness. Age was not associated with perceived pros and cons of smoking. 


\section{Qualitative Findings}

The qualitative studies supported the quantitative findings. Esterberg and Perneger (55) reported that people living with schizophrenia smoke to relax, gain relief from negative symptoms, and relieve boredom. Similar to Spring et al. (54), Esterberg and Perneger (55) reported that the majority of participants believed there were more pros to smoking than cons. Lawn et al. (56) found that people living with schizophrenia expressed little concern for their physical health, preferring to smoke as a way to manage positive symptoms and improve problem solving skills. In contrast, Esterberg and Perneger (55) reported that people living with schizophrenia were aware of the negative health implications, including cancer, and reduced engagement in physical activity, and the financial burden of smoking. Health concerns and a desire to increase selfesteem prompted eleven of the twelve participants to attempt quitting, yet feelings of tension and nervousness led them to begin smoking again. Two studies reported that people living with schizophrenia felt that their family, friends, and health professionals provided little reinforcement for them to quit $(55,56)$.

Following cravings as the most commonly reported barrier to smoking cessation (53\%), Goldberg et al. (57) reported that pleasure and enjoyment associated with smoking, as well as coping with symptoms of anxiety were both reported as barriers to quitting by $20 \%$ of people living with schizophrenia. Habit (19\%), boredom (17\%), and a social environment associated with pressure to smoke and that provided little support to quit (13\%) were other important barriers to smoking cessation. Other barriers reported in the qualitative studies were that people living with schizophrenia smoke for a sense of identity as it has shaped their development and contributes to their current sense of self and to feel freedom from their powerlessness in deciding their future (56). People living with schizophrenia also reported that the lack of smoking cessation programs in hospitals was also a barrier to quitting (55). People living with schizophrenia generally viewed NRT as negative and associated NRT with a sense of increasing rather than decreasing cravings, being unhealthy, unwanted side-effects and viewed NRT as unnecessary to quit (55). Participants believed that reducing, rather than quitting, would be a more realistic goal (56).

\section{DISCUSSION}

We systematically reviewed barriers to smoking cessation in 23 studies including 3,257 people living with schizophrenia. People living with schizophrenia reported that the main reasons for smoking are to manage cravings and addiction, as well as negative symptoms such as their desire to reduce negative affect, facilitate social relationships, manage stress, and relieve boredom. Medical professionals may be less likely to provide smoking cessation advice to people living with schizophrenia when compared with people without mental illness. The social networks of people living with schizophrenia may show little support for smoking cessation, which may reduce the likelihood people living with schizophrenia will attempt to quit smoking. While people living with schizophrenia appeared to be as aware of the smokingrelated health risks as people without mental illness, they may be less likely to act on this awareness. Overall, it appears that people living with schizophrenia experience a greater number of barriers to smoking cessation than people without mental illness.

Cravings and addiction to smoking were reported as one of the main reasons for smoking in people living with schizophrenia in five studies $(35-38,57)$. The highly addictive properties of nicotine may have a greater influence on people living with schizophrenia compared with those without mental illness (36, 40, 41). Past research suggests that people living with schizophrenia have a higher nicotine dependence than people in the general population (1). These findings indicate the need to address the physical addiction to smoking with NRT, such as patches or gum, or smoking cessation medications, such as varenicline or bupropion.

While pharmacotherapy may increase smoking cessation rates, one qualitative study included in this review highlighted the strong negative attitudes that people living with schizophrenia have toward NRT, such as the belief that NRT increased cravings rather than decreased them, that NRT made them feel sick and that NRT was unhealthy (55). Negative attitudes toward NRT are also common among smokers without schizophrenia $(58,59)$. These findings are concerning, as we identified one study examining people living with schizophrenia which found that positive attitudes toward NRT held the strongest relationship with greater intention to use NRT (53). Attitudes toward pharmacotherapy may be an important psychosocial component of combined pharmacological and psychosocial interventions. Further intervention research examining the role of psychosocial support in promoting adherence to pharmacological treatments, including NRT, in people living with schizophrenia is required.

People living with schizophrenia also reported smoking to reduce negative affect, relieve boredom, manage stress, and facilitate social relationships. These reasons for smoking all target negative symptoms of schizophrenia, supporting previous research that has identified a link between greater negative symptom severity and increased smoking rates or nicotine dependence $(2,60,61)$. However, in contrast to the views of people living with schizophrenia, smoking cessation is associated with lower levels of stress, anxiety, and depression than continued smoking (62). Psychoeducation on the effects of smoking on negative symptoms and other psychological treatment to manage negative symptoms may help reduce smoking rates in people living with schizophrenia. The National Institute for Health Care and Excellence (NICE) guidelines recommend CBT as a psychological treatment for schizophrenia (63). A 2014 metaanalysis found that psychological treatments, such as CBT, and pharmacological treatments, such as antidepressants and second-generation antipsychotics, significantly reduced negative symptoms in people living with schizophrenia (64).

Only one study has examined CBT to promote smoking cessation in people living with schizophrenia, which found significantly higher abstinence and reduction rates at 3, 6, and 12 months in participants who attended all 8 treatment sessions when compared with participants receiving treatment as usual (8). Continued research examining CBT on smoking cessation 
in people living with schizophrenia is required. Behavioral activation, an important component of CBT, is an effective standalone treatment for depression and may have a unique influence on negative symptoms of schizophrenia $(65,66)$, however no research examining the effect of behavioral activation on smoking cessation has been conducted in people living with schizophrenia. Future research examining the mediating role of negative symptoms in treatment effect on smoking cessation will enhance the evidence linking smoking with negative symptoms.

Most people living with schizophrenia appear to have some awareness of the health risks associated with smoking (36, $40,43,44)$ and health risks are often cited as a facilitator to quit smoking $(35,38)$. Health concerns are also considered the most important reason to quit smoking among the general population $(25,67)$. Health professionals should continue to raise awareness of the health risks of smoking among people living with schizophrenia. A review found that half of the reviewed interventions to improve health literacy among primary care patients led to reduced smoking rates, with significant outcomes most commonly associated with information that was provided via individual counseling or through written resources (68).

Considering our findings that people living with schizophrenia rate health risks as an important reasons to quit smoking, the authors strongly encourage health professionals to follow the 5 A's (ask, assess, advice, assist, arrange) when working with people living with schizophrenia. Unfortunately, we found mixed evidence from the seven studies examining receipt of advice to quit from a physician. Two studies found that people living with schizophrenia were less likely to be advised to quit smoking compared with people without mental illness $(48,51)$, while a third non-comparison study found that only $25 \%$ of people living with schizophrenia were advised to quit (46). Barriers impeding clinicians' likelihood of administering smoking cessation advice to people with mental illness included a perceived lack of interest to quit in patients and insufficient staff and time to provide such support (52). Training that aims to improve physicians' attitudes and perceived competence to deliver the 5 A's may increase their likelihood of delivering smoking cessation advice, especially when training is combined with other interventions components, such as patient counseling, patient access to tailored information resources, and free NRT (69).

\section{Strengths and Limitations}

Our review amalgamated a diverse range of studies that included individuals from a variety of geographical locations and community or clinical settings, thereby enhancing the generalizability of the findings. However, we failed to identify eligible articles from Asia, South America, or Africa. Thus, our findings may be limited to people living with schizophrenia residing in Western societies.

We limited our review to include samples in which people living with schizophrenia were the majority. Six studies included a mixed sample of people living with schizophrenia or other mental illnesses, yet $91.6 \%$ of participants in groups including people living with schizophrenia were diagnosed with schizophrenia. Thus, we can be confident that our findings are specific to the target population and that they overcome limitations of previous reviews that combine results for people living with severe mental illness, such as bipolar disorder and severe depression.

We chose to include articles that contained baseline data from intervention studies. In doing so, we increased the evidence base and quality of available evidence. Yet, we may have biased the sample by including studies that excluded people living with schizophrenia who were ineligible to participate in the intervention. Common exclusion criteria of intervention studies included in our review were cognitive impairments, diagnosis of a medical condition precluding use of NRT, or brain injury. Our findings may not extend to people living with schizophrenia with these comorbidities.

The 23 articles included in our study held a number of limitations that may also affect the reliability of our findings. Nine studies (39\%) included samples with fewer than 50 participants and eight studies (35\%) had sample sizes between 51 and 100 . Small sample sizes reduce the reliability and generalizability of the findings. Six of the $20(30 \%)$ quantitative studies did not include a control sample limiting our understanding of whether the findings were different for people without schizophrenia or other mental illnesses. We found that there was inconsistent use of validated assessment tools. Three studies used the Reasons For Quitting scale (70), two used the Reasons for Smoking Questionnaire (71) and two used the Smoking Consequences Questionnaire (72). Most other studies used assessment tools purposively developed for their study. The reliability of evidence will be improved with more studies using the same assessment tool. Future research may also wish to validate these assessment tools in people living with schizophrenia and should continue to explore reasons for smoking and quitting that are not addressed in these assessment tools.

\section{Recommendations for Future Research and Practice}

Our review highlighted the presence of a number of important psychosocial barriers and facilitators to smoking cessation in people living with schizophrenia. A 2013 Cochrane review found only five randomized controlled trials examining psychosocial intervention effects on smoking cessation in people living with schizophrenia (7). Our findings can be used to inform the development of psychosocial interventions that are combined with pharmacological treatments. Future research that examines the effects of separate treatment components (e.g., cognitive vs. behavioral components of CBT) and the role of possible mediators, such as reduced negative symptoms or baseline nicotine dependence, will continue to inform the literature of the mechanisms facilitating smoking cessation among people living with schizophrenia.

Our findings strongly support current smoking cessation guidelines for physicians to advise people living with schizophrenia that cravings, withdrawal symptoms, nicotine dependence, and health risks will continue if they continue to smoke (73). Physicians may also assist people living with schizophrenia quit smoking by providing information on 
smoking-related health risks, delivering brief counseling or MI, or referring patients to specialized smoking cessation services. Future research may be required to examine strategies to overcome barriers reducing physicians' likelihood of providing advice and assistance, such as through brief professional training programs. In addition to increasing physicians' likelihood of providing advice and assistance, future research should assess the impact of information resources educating people living with schizophrenia of the smoking-related health risks on their motivation to quit smoking and smoking cessation rates.

While the evidence is far from conclusive, electronic nicotine devices (i.e., e-cigarettes) may help reduce smoking rates by managing both the physical and psychological addictive properties of cigarettes (74). The regulation of e-cigarettes and nicotine liquids for use in e-cigarettes varies worldwide. In some countries, nicotine liquids cannot be purchased without a prescription. Ongoing research in this area is needed to help determine whether e-cigarettes increase smoking cessation rates, whether people living with schizophrenia are open to using e-cigarettes, and how physicians may influence the uptake of e-cigarettes for smoking cessation purposes.

\section{CONCLUSION}

Our systematic review found a range of important barriers and facilitators to smoking cessation in people living with schizophrenia. Addiction and cravings appear to be a primary

\section{REFERENCES}

1. de Leon J, Diaz FJ. A meta-analysis of worldwide studies demonstrates an association between schizophrenia and tobacco smoking behaviors. Schizophr Res. (2005) 76:135-57. doi: 10.1016/j.schres.2005.02.010

2. Cooper J, Mancuso SG, Borland R, Slade T, Galletly C, Castle D. Tobacco smoking among people living with a psychotic illness: the second Australian Survey of Psychosis. Aust N Z J Psychiatry (2012) 46:851-63. doi: 10.1177/0004867412449876

3. Hausswolff-Juhlin V, Bjartveit M, Lindström E, Jones P. Schizophrenia and physical health problems. Acta Psychiatr Scand. (2009) 119:15-21. doi: 10.1111/j.1600-0447.2008.01309.x

4. Fiore MC, Jaen CR, Baker T, Bailey W, Benowitz N, Curry SE, et al. Treating Tobacco Use and Dependence: 2008 Update. Rockville, MD: US Department of Health and Human Services (2008).

5. Zwar N, Richmond R, Borland R, Peters M, Litt J, Bell J, et al. Supporting Smoking Cessation: A Guide for Health Professionals. Melbourne, VIC: The Royal Australian College of General Practitioners (2011).

6. Mendelsohn CP, Kirby DP, Castle DJ. Smoking and mental illness. An update for psychiatrists. Australas Psychiatry (2015) 23:37-43. doi: $10.1177 / 1039856214562076$

7. Tsoi DT, Porwal M, Webster AC. Interventions for smoking cessation and reduction in individuals with schizophrenia. Cochrane Database Syst Rev. (2013) 2:Cd007253. doi: 10.1002/14651858.CD007253.pub3

8. Baker A, Richmond R, Haile M, Lewin TJ, Carr VJ, Taylor RL, et al. A randomized controlled trial of a smoking cessation intervention among people with a psychotic disorder. Am J Psychiatry (2006) 163:1934-42. doi: 10.1176/ajp.2006.163.11.1934

9. Gallagher SM, Penn PE, Schindler E, Layne W. A comparison of smoking cessation treatments for persons with schizophrenia and other serious mental illnesses. J. Psychoactive Drugs (2007) 39:487-97. doi: 10.1080/02791072.2007.10399888 reason why people living schizophrenia smoke cigarettes, yet there is also strong evidence that they smoke to manage features of negative symptoms, such as stress, negative affect, boredom, and social isolation. Health professionals also play an important role in smoking cessation for people living with schizophrenia and should support quitting in people living with schizophrenia as much as they do for people without mental illness. The barriers and facilitators identified in this review should be used to inform the development of targeted psychosocial components of smoking cessation interventions for people living with schizophrenia.

\section{AUTHOR CONTRIBUTIONS}

$\mathrm{AL}, \mathrm{ES}, \mathrm{OW}$, and $\mathrm{BB}$ contributed to the study conception and design. AL, ES, and OW collected, analyzed, and interpreted data. $\mathrm{AL}$ wrote the first draft of the manuscript. AL, ES, OW, and $\mathrm{BB}$ contributed to critical revision of the manuscript, read and approved the submitted version.

\section{FUNDING}

BB was funded by a National Health and Medical Research Council (NHMRC) Career Development Fellowship (1063206) and a Faculty of Health and Medicine, University of Newcastle, Faculty of Health and Medicine Gladys M Brawn Career Development Fellowship.

10. Tidey JW, Rohsenow DJ, Kaplan GB, Swift RM, Reid N. Effects of contingency management and bupropion on cigarette smoking in smokers with schizophrenia. Psychopharmacology (2011) 217:279-87. doi: 10.1007/s00213-011-2282-8

11. George TP, Ziedonis DM, Feingold A, Pepper WT, Satterburg CA, Winkel J, et al. Nicotine transdermal patch and atypical antipsychotic medications for smoking cessation in schizophrenia. Am J Psychiatry (2000) 157:1835-42. doi: 10.1176/appi.ajp.157.11.1835

12. Steinberg ML, Ziedonis DM, Krejci JA, Brandon TH. Motivational interviewing with personalized feedback: a brief intervention for motivating smokers with schizophrenia to seek treatment for tobacco dependence. J Consult Clin Psychol. (2004) 72:723. doi: 10.1037/0022-006X.72. 4.723

13. Williams J, Steinberg ML, Zimmermann MH, Gandhi KK, Stipelman B, Budsock PD, et al. Comparison of two intensities of tobacco dependence counseling in schizophrenia and schizoaffective disorder. J Subst Abuse Treat. (2010) 38:384-93. doi: 10.1016/j.jsat.2010.03.006

14. Gelkopf M, Noam S, Rudinski D, Lerner A, Behrbalk P, Bleich A, et al. Nonmedication smoking reduction program for inpatients with chronic schizophrenia: a randomized control design study. J Nerv Ment Dis. (2012) 200:142-6. doi: 10.1097/NMD.0b013e3182438e92

15. Addington J, El-Guebaly N, Addington D, Hodgins D. Readiness to stop smoking in schizophrenia. Can J Psychiatry (1997) 42:49-52. doi: 10.1177/070674379704200107

16. George TP, Vessicchio JC, Termine A, Bregartner TA, Feingold A, Rounsaville BJ, et al. A placebo controlled trial of bupropion for smoking cessation in schizophrenia. Biol Psychiatry (2002) 52:53-61. doi: 10.1016/S0006-3223(02)01339-2

17. George TP, Vessicchio JC, Sacco KA, Weinberger AH, Dudas MM, Allen TM, et al. A placebo-controlled trial of bupropion combined with nicotine patch for smoking cessation in schizophrenia. Biol Psychiatry (2008) 63:1092-6. doi: 10.1016/j.biopsych.2007.11.002 
18. Williams JM, Anthenelli RM, Morris CD, Treadow J, Thompson JR, Yunis $\mathrm{C}$, et al. A randomized, double-blind, placebo-controlled study evaluating the safety and efficacy of varenicline for smoking cessation in patients with schizophrenia or schizoaffective disorder. J Clin Psychiatry (2012) 73:654-60. doi: 10.4088/JCP.11m07522

19. Wing VC, Wass CE, Soh DW, George TP. A review of neurobiological vulnerability factors and treatment implications for comorbid tobacco dependence in schizophrenia. Ann N Y Acad Sci. (2012) 1248:89-106. doi: 10.1111/j.1749-6632.2011.06261.x

20. Ziedonis DM, George TP. Schizophrenia and nicotine use: report of a pilot smoking cessation program and review of neurobiological and clinical issues. Schizophr Bull. (1997) 23:247. doi: 10.1093/schbul/23.2.247

21. Steinberg ML, Williams J. Psychosocial treatments for individuals with schizophrenia and tobacco dependence. J Dual Diagn. (2007) 3:99-112. doi: 10.1300/J374v03n03_08

22. Goldberg JO. Successful change in tobacco use in schizophrenia. J Am Psychiatr Nurses Assoc. (2010) 16:30-5. doi: 10.1177/1078390309357084

23. Trainor K, Leavey G. Barriers and facilitators to smoking cessation among people with severe mental illness: a critical appraisal of qualitative studies. Nicotine Tob Res. (2017) 19:14-23. doi: 10.1093/ntr/ntw183

24. Twyman L, Bonevski B, Paul C, Bryant J. Perceived barriers to smoking cessation in selected vulnerable groups: a systematic review of the qualitative and quantitative literature. BMJ Open (2014) 4:e006414. doi: 10.1136/bmjopen-2014-006414

25. McCaul KD, Hockemeyer JR, Johnson RJ, Zetocha K, Quinlan K, Glasgow RE. Motivation to quit using cigarettes: a review. Addict Behav. (2006) 31:42-56. doi: 10.1016/j.addbeh.2005.04.004

26. Winterer G. Why do patients with schizophrenia smoke? Curr Opin Psychiatry (2010) 23:112-9. doi: 10.1097/YCO.0b013e3283366643

27. Milev P, Ho B-C, Arndt S, Andreasen NC. Predictive values of neurocognition and negative symptoms on functional outcome in schizophrenia: a longitudinal first-episode study with 7-year follow-up. Am J Psychiatry (2005) 162:495-506. doi: 10.1176/appi.ajp.162.3.495

28. Ziedonis DM, Hitsman B, Beckham JC, Zvolensky M, Adler LE, AudrainMcGovern J, et al. Tobacco use and cessation in psychiatric disorders: national institute of mental health report. Nicotine Tob Res. (2008) 10:1691-715. doi: 10.1080/14622200802443569

29. de Leon J, Gurpegui M, Diaz F. Epidemiology of comorbid tobacco use and schizophrenia: thinking about risks and protective factors. J Dual Diagn. (2007) 3:9-25. doi: 10.1300/J374v03n03_03

30. Morisano D, Bacher I, Audrain-McGovern J, George TP. Mechanisms underlying the comorbidity of tobacco use in mental health and addictive disorders. Can J Psychiatry (2009) 54:356-67. doi: 10.1177/070674370905400603

31. Rae J, Pettey D, Aubry T, Stol J. Factors affecting smoking cessation efforts of people with severe mental illness: a qualitative study. J Dual Diagn. (2015) 11:42-9. doi: 10.1080/15504263.2014.992096

32. Moher D, Liberati A, Tetzlaff J, Altman DG, Group P. Preferred reporting items for systematic reviews and meta-analyses: the PRISMA statement. PLoS Med. (2009) 6:e1000097. doi: 10.1371/journal.pmed.1000097

33. Covidence systematic review software. Veritas Health Innovation. (Melbourne, VIC)

34. Kmet LM, Cook LS, Lee RC. Standard Quality Assessment Criteria for Evaluating Primary Research Papers From a Variety of Fields (2004).

35. Filia SL, Baker AL, Richmond R, Castle DJ, Kay-Lambkin FJ, Sakrouge R, et al. Health behaviour risk factors for coronary heart disease (CHD) in smokers with a psychotic disorder: baseline results. Mental Health Subst Use Dual Diagn. (2011) 4:158-71. doi: 10.1080/17523281.2011.555088

36. Tulloch HE, Pipe AL, Clyde MJ, Reid RD, Els C. The quit experience and concerns of smokers with psychiatric illness. Am J Prev Med. (2016) 50:70918. doi: 10.1016/j.amepre.2015.11.006

37. Forchuk C, Norman R, Malla A, Martin ML, McLean T, Cheng S, et al. Schizophrenia and the motivation for smoking. Perspect Psychiatr Care (2002) 38:41-9. doi: 10.1111/j.1744-6163.2002.tb00656.x

38. Mann-Wrobel MC, Bennet ME, Weiner EE, Buchanan RW, Ball MP. Smoking history and motivation to quit in smokers with schizophrenia in a smoking cessation program. Schizophr Res. (2011) 126:277-83. doi: $10.1016 /$ j.schres.2010.10.030
39. Tanriover O, Karamustafalioglu N, Tezvaran Z, Kaplan A, Tomruk N Frequency of cigarette smoking among psychiatric inpatients evaluated by the Fagerstrom test for nicotine dependence. Asian Pac J Cancer Prev. (2013) 14:579-82. doi: 10.7314/APJCP.2013.14.1.579

40. Baker A, Richmond R, Haile M, Lewin TJ, Carr VJ, Taylor RL, et al. Characteristics of smokers with a psychotic disorder and implications for smoking interventions. Psychiatry Res. (2007) 150:141-52. doi: 10.1016/j.psychres.2006.05.021

41. Tidey JW, Colby SM, Xavier EMH. Effects of smoking abstinence on cigarette craving, nicotine withdrawal, and nicotine reinforcement in smokers with and without schizophrenia. Nicotine Tob Res. (2014) 16:326-34. doi: $10.1093 / \mathrm{ntr} / \mathrm{ntt} 152$

42. Kelly DL, Raley HG, Lo S, Wright K, Liu F, McMahon RP, et al. Perception of smoking risks and motivation to quit among nontreatment-seeking smokers with and without schizophrenia. Schizophr Bull. (2012) 38:543-51. doi: $10.1093 /$ schbul/sbq124

43. Filia SL, Baker AL, Gurvich CT, Richmond R, Kulkarni J. The perceived risks and benefits of quitting in smokers diagnosed with severe mental illness participating in a smoking cessation intervention: gender differences and comparison to smokers without mental illness. Drug Alcohol Rev. (2014) 33:78-85. doi: 10.1111/dar.12091

44. Tidey JW, Rohsenow DJ. Smoking expectancies and intention to quit in smokers with schizophrenia, schizoaffective disorder and non-psychiatric controls. Schizophr Res. (2009) 115:310-6. doi: 10.1016/j.schres.2009. 09.032

45. Krishnadas R, Jauhar S, Telfer S, Shivashankar S, McCreadie RG. Nicotine dependence and illness severity in schizophrenia. Br J Psychiatry (2012) 201:306-12. doi: 10.1192/bjp.bp.111.107953

46. Kourakos M, Koukia E. A study of patients' smoking habits in a psychiatric hospital. Int J Car Sci. (2014) 7:592-601.

47. Coletti DJ, Brunette M, John M, Kane JM, Malhotra AK, Robinson DG. Responses to tobacco smoking-related health messages in young people with recent-onset schizophrenia. Schizophr Bull. (2015) 41:1256-65. doi: $10.1093 / \mathrm{schbul} / \mathrm{sbv} 122$

48. Briskman I, Bar G, Boaz M, Shargorodsky M. Impact of co-morbid mental illness on the diagnosis and management of patients hospitalized for medical conditions in a general hospital. Int J Psychiatry Med. (2012) 43:339-48. doi: 10.2190/PM.43.4.d

49. Hippisley-Cox JP, Parker C, Coupland C, Vinogradova Y. Inequalities in the primary care of patients with coronary heart disease and serious mental health problems: a cross-sectional study. Heart (2007) 93:1256-62. doi: $10.1136 /$ hrt.2006.110171

50. Himelhoch S, Leith J, Goldberg R, Kreyenbuhl J, Medoff D, Dixon L. Care and management of cardiovascular risk factors among individuals with schizophrenia and type 2 diabetes who smoke. Gen Hosp Psychiatry (2009) 31:30-2. doi: 10.1016/j.genhosppsych.2008.07.007

51. Duffy SA, Kilbourne AM, Austin KL, Dalack GW, Woltmann EM, Waxmonsky J, et al. Risk of smoking and receipt of cessation services among veterans with mental disorders. Psychiatr Serv. (2012) 63:325-32. doi: 10.1176/appi.ps.201100097

52. Brown CH, Medoff D, Dickerson FB, Fang LJ, Lucksted A, Goldberg RW, et al. Factors influencing implementation of smoking cessation treatment within community mental health centers. J Dual Diagn. (2015) 11:145-50. doi: 10.1080/15504263.2015.1025025

53. Brunette MF, Feiron JC, Aschbrenner K, Colctti D, Devitt T, Greene MA, et al. Characteristics and predictors of intention to use cessation treatment among smokers with schizophrenia: young adults compared to older adults. J Subst Abus Alcohol. (2017) 5:1055.

54. Spring B, Pingitore R, McChargue DE. Reward value of cigarette smoking for comparably heavy smoking schizophrenic, depressed, and nonpatient smokers. Am J Psychiatry (2003) 160:316-22. doi: 10.1176/appi.ajp.160.2.316

55. Esterberg ML, Compton MT. Smoking behavior in persons with a schizophrenia-spectrum disorder: a qualitative investigation of the transtheoretical model. Soc Sci Med. (2005) 61:293-303. doi: 10.1016/j.socscimed.2004.11.057

56. Lawn SJ, Pols RG, Barber JG. Smoking and quitting: a qualitative study with community-living psychiatric clients. Soc Sci Med. (2002) 54:93-104. doi: 10.1016/S0277-9536(01)00008-9 
57. Goldberg JO, Moll S, Washington A. Exploring the challenge of tobacco use and schizophrenia. Psychiatr Rehab Skills (1996) 1:51-63. doi: 10.1080/10973435.1996.10387541

58. Etter JF, Perneger TV. Attitudes toward nicotine replacement therapy in smokers and ex-smokers in the general public. Clin Pharmacol Ther. (2001) 69:175-83. doi: 10.1067/mcp.2001.113722

59. Uppal N, Shahab L, Britton J, Ratschen E. The forgotten smoker: a qualitative study of attitudes towards smoking, quitting, and tobacco control policies among continuing smokers. BMC Public Health (2013) 13:432. doi: 10.1186/1471-2458-13-432

60. Patkar AA, Gopalakrishnan R, Lundy A, Leone FT, Certa KM, Weinstein SP. Relationship between tobacco smoking and positive and negative symptoms in schizophrenia. J Nerv Ment Dis. (2002) 190:604-10. doi: 10.1097/00005053-200209000-00005

61. Saliba M, Assaad S, Haddad C, Hallit S, Hachem D, Haddad G. Schizophrenia and smoking: impact on negative symptoms. Revista Internacional Investig Adicciones (2017) 3:28-35. doi: 10.28931/riiad.2017.1.04

62. Taylor G, McNeill A, Girling A, Farley A, Lindson-Hawley N, Aveyard P. Change in mental health after smoking cessation: systematic review and meta-analysis. BMJ (2014) 348:g1151. doi: 10.1136/bmj.g1151

63. NICE (2014). Psychosis and Schizophrenia in Adults: Prevention and Management. NICE.

64. Fusar-Poli P, Papanastasiou E, Stahl D, Rocchetti M, Carpenter W, Shergill $\mathrm{S}$, et al. Treatments of negative symptoms in schizophrenia: meta-analysis of 168 randomized placebo-controlled trials. Schizophr Bull. (2014) 41:892-9. doi: $10.1093 /$ schbul/sbu170

65. Mairs H, Lovell K, Campbell M, Keeley P. Development and pilot investigation of behavioral activation for negative symptoms. Behav Modif. (2011) 35:486-506. doi: 10.1177/0145445511411706

66. Choi K-H, Jaekal E, Lee G-Y. Motivational and behavioral activation as an adjunct to psychiatric rehabilitation for mild to moderate negative symptoms in individuals with schizophrenia: a proof-of-concept pilot study. Front Psychol. (2016) 7:1759. doi: 10.3389/fpsyg.2016.01759

67. Curry SJ, Grothaus L, McBride C. Reasons for quitting: intrinsic and extrinsic motivation for smoking cessation in a population-based sample of smokers. Addict Behav. (1997) 22:727-39. doi: 10.1016/S0306-4603(97)00059-2
68. Taggart J, Williams A, Dennis S, Newall A, Shortus T, Zwar N, et al. A systematic review of interventions in primary care to improve health literacy for chronic disease behavioral risk factors. BMC Fam Pract. (2012) 13:49. doi: 10.1186/1471-2296-13-49

69. Papadakis S, McDonald P, Mullen K-A, Reid R, Skulsky K, Pipe A. Strategies to increase the delivery of smoking cessation treatments in primary care settings: a systematic review and meta-analysis. Prev Med. (2010) 51:199-213. doi: 10.1016/j.ypmed.2010.06.007

70. Curry S, Wagner EH, Grothaus LC. Intrinsic and extrinsic motivation for smoking cessation. J Consult Clin Psychol. (1990) 58:310. doi: 10.1037/0022-006X.58.3.310

71. Pederson LL, Bull SB, Ashley MJ, MacDonald JK. Quitting smoking: why, how, and what might help. Tob Control (1996) 5:209-14. doi: 10.1136/tc.5. 3.209

72. Copeland AL, Brandon TH, Quinn EP. The smoking consequences questionnaire-adult: measurement of smoking outcome expectancies of experienced smokers. Psychol Assess. (1995) 7:484. doi: 10.1037/1040-3590.7.4.484

73. RACGP (2011). Supporting Smoking Cessation: A Guide for Health Professionals. Melbourne, VIC: The Royal Australian College of General Practitioners.

74. Schivo M, Avdalovic MV, Murin S. Electronic nicotine delivery systems for smoking cessation: where are we? Curr Respir Care Rep. (2014) 3:192-9. doi: 10.1007/s13665-014-0088-y

Conflict of Interest Statement: The authors declare that the research was conducted in the absence of any commercial or financial relationships that could be construed as a potential conflict of interest.

Copyright (C) 2018 Lum, Skelton, Wynne and Bonevski. This is an open-access article distributed under the terms of the Creative Commons Attribution License (CC BY). The use, distribution or reproduction in other forums is permitted, provided the original author(s) and the copyright owner(s) are credited and that the original publication in this journal is cited, in accordance with accepted academic practice. No use, distribution or reproduction is permitted which does not comply with these terms. 